\title{
An Efficient Method of Image Denoising Using Hybrid Filter Approach
}

\author{
Deepa Detani, Dr.Akhilesh Upadhyay, Dr.Meenal Saxena
}

\begin{abstract}
Image degradation generally occurs due to transmission channel error, camera misfocus, atmospheric turbulence, relative object-camera motion, etc. Such degradations are unavoidable while a scene is captured through a camera. As degraded images are having less scientific values, restoration of such images is extremely essential in many practical applications.

Visual information transmitted in the form of digital images is becoming a major method of communication in the modern age, but the image obtained after transmission is often corrupted with noise. The received image needs processing before it can be used in applications. Image denoising involves the manipulation of the image data to produce a visually high quality image. Salt \& pepper (Impulse) noise and the additive white Gaussian noise and blurredness are the types of noise that occur during transmission and capturing. To remove these types of noise we have many filters like mean filter, median filter, inverse filter, wiener filter. No single filter can remove both type of noise.

In this paper, attempts have been made to recover images from their degraded observations. Various degradations including, Gaussian noise and salt-n-pepper has been considered, and to recover the image hybrid filter approach has been proposed.

Keywords: Image Denoising, Salt \& pepper noise, Gaussian noise, Pseudo inverse filter, Weiner filter
\end{abstract}

\section{Introduction:}

Visual information transmitted in the form of digital images is becoming a major method of communication in the modern age, but the image obtained after transmission is often corrupted with noise. The received image needs processing before it can be used in applications. Image denoising involves the manipulation of the image data to produce a visually high quality image. This paper gives us a brief idea about various image denoising methods. The numerical computation has been done using MATLAB 7.8.0.

Image denoising is often used in the field of photography or publishing where an image was somehow degraded but needs to be improved before it can be printed. For this type of application we need to know something about the degradation process in order to develop a model for it. When we have a model for the degradation process, the inverse process can be applied to the image to restore it back to the original form.

\section{Types of Noises}

Noise in image is caused by fluctuations in the brightness or colour information at the pixels. Noise is a process which distorts the acquired image and is not a part of the original image. Noise in images can occur in many ways. During image acquisition the optical signals get converted into electrical which then gets converted to digital signal. At each process of conversion noise gets added to the image. The image can also become noisy during transmission of the image in the form of digital signals. The types of noises are:

1. Gaussian noise

3. Shot noise (Poisson noise)
2. Salt and Pepper noise

4. Speckle noise

\section{Research \& Development in various Image Denoising Techniques}

An image is often corrupted by noise in its acquisition and transmission. Image denoising is used to remove the additive noise while retaining as much as possible the important signal features. In the recent years there has been a fair amount of research on wavelet thresholding and threshold selection for signal de-noising [1],[2], because wavelet provides an appropriate basis for separating noisy signal from the image signal. The motivation is that as the wavelet transform is good at energy compaction, the small coefficient is more likely due to noise and large coefficient due to important signal features. These small coefficients can be thresholded without affecting the significant features of the image.

Thresholding is a simple non-linear technique, which operates on one wavelet coefficient at a time. In its most basic form, each coefficient is thresholded by comparing against threshold, if the coefficient is smaller than threshold, set to zero;otherwise it is kept or modified. Replacing the small noisy coefficients by zero and inverse wavelet transform on the result may lead to reconstruction with the essential signal characteristics and with less noise. 
Other methods include filtering approach Objectives of any filtering approach are:

- To suppress the noise effectively in uniform regions.

- To preserve edges and other similar image characteristics.

- To provide a visually natural appearance . [3]

\section{Proposed method}

The basic idea behind this paper is the estimation of the uncorrupted image from the distorted or noisy image, and is also referred to as image "denoising". There are various methods to help restore an image from noisy distortions. Selecting the appropriate method plays a major role in getting the desired image. The denoising methods tend to be problem specific. For example, a method that is used to denoise satellite images may not be suitable for denoising medical images. In this paper, a hybrid approach is used to carry out image denoising \& is implemented in Matlab 6.1 [4]. The hybrid approach is based on Weiner filter \& Pseudo inverse filter. First of all performance of individual filter in terms of Signal to Noise Ratio (SNR) is computed and then both the filters are taken as one hybrid filter. The Signal to Noise Ratio (SNR) of both the approaches are then compared. In order to quantify the performance of the various denoising algorithms, a high quality image is taken from MATLAB environment and some known noise is added to it. This would then be given as input to the denoising algorithm, which produces an image close to the original high quality image. The performance of each algorithm is compared by computing Signal to Noise Ratio (SNR) besides the visual interpretation.

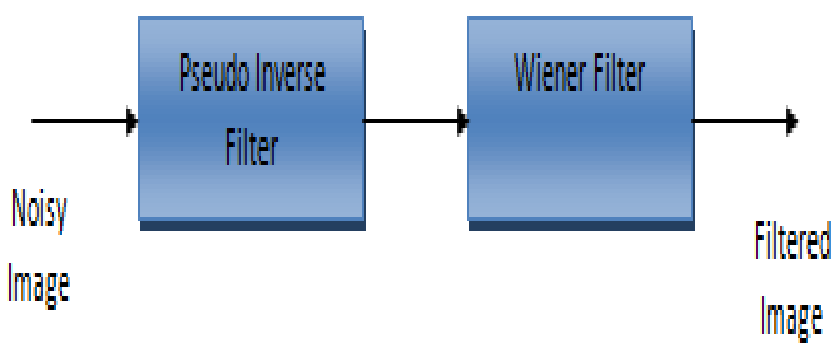

Fig 4.1 Hybrid Filter

\section{Implementation\& Simulation}

As we have already studied a number of noises and to remove them there is a quite good category of filters available as:

- Pseudo Inverse Filter Filter

- Wiener Filter

- Inverse Filter and many more

But to remove maximum possible noise from the signal is not possible with any single filter. So, we have found a new approach for de noising based on multi level filtering.

Our filter is a combination of: Pseudo Inverse Filter Filter Wiener Filter

\section{Pseudo Inverse Filters}

Suppose we have a known image function $\mathrm{f}(\mathrm{x}, \mathrm{y})$ and a blurring function $\mathrm{h}(\mathrm{x}, \mathrm{y})$, so we need to recover $\mathrm{f}(\mathrm{x}, \mathrm{y})$ from the convolution

$$
\mathrm{g}(\mathrm{x}, \mathrm{y})=\mathrm{f}(\mathrm{x}, \mathrm{y}) * \mathrm{~h}(\mathrm{x}, \mathrm{y})-------\mathrm{EQ}(1)
$$

A very easy possible solution for this is INVERSE FILTERING.

$\mathrm{EQ}(1)$ can be written in frequency domain as:

$$
\mathrm{G}(\mathrm{u}, \mathrm{v})=\mathrm{F}(\mathrm{u}, \mathrm{v}) \times \mathrm{H}(\mathrm{u}, \mathrm{v})--------\mathrm{EQ}(2)
$$

Now we divide eq (2) by $\mathrm{H}(\mathrm{u}, \mathrm{v})$ and so is referred to as Inverse Filtering

This can be written as:

$$
\mathrm{G}(\mathrm{u}, \mathrm{v}) / \mathrm{H}(\mathrm{u}, \mathrm{v})=\{\mathrm{F}(\mathrm{u}, \mathrm{v}) \times \mathrm{H}(\mathrm{u}, \mathrm{v})\} / \mathrm{H}(\mathrm{u}, \mathrm{v})-------\mathrm{EQ}(3)
$$

$$
\mathrm{G}(\mathrm{u}, \mathrm{v}) / \mathrm{H}(\mathrm{u}, \mathrm{v})=\mathrm{F}(\mathrm{u}, \mathrm{v})--------\mathrm{EQ}(4)
$$

These filters are very effective on removing White Gaussian Noise. 


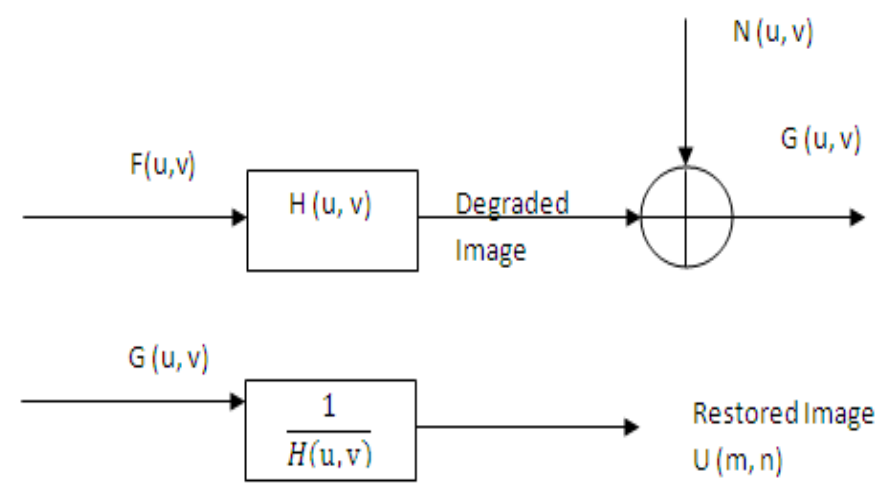

Fig 5.1 Block Diagram of Pseudo Inverse Filter

\section{Wiener Filter}

These filters reduces the amount of noise present in the signal by comparison with an estimation of a desired noiseless signal.

It is based on Statistical Approach.[8]

Here, we assume to have knowledge of the spectral properties of the original signal and the noise and seek the Linear Time Invariant Filter whose output would be as close to the original signal as possible.

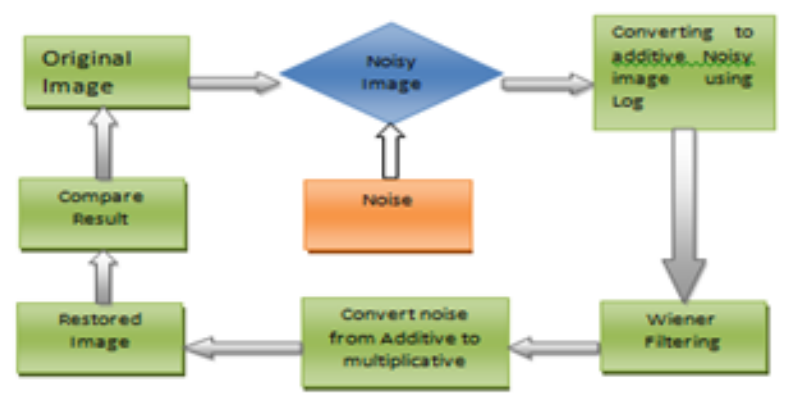

Fig 5.2 Flowchart of Denoising algorithm using Weiner filter

\section{Results \& comparison}

This method assumes that the discontinuities in an image are only due to noise. For this reason, all the experiments are done on an image with very little variation in intensity. A test image where all pixel values having a magnitude of 100 is created and noise is added to it with the imnoise( ) function. Denoising is carried out using individual filter first \& then the combination and Signal to Noise Ratio (SNR) [7] for each of these outputs is computed.

The SNR is defined as

$\mathrm{SNR}=20 \log _{10}\left(\frac{a_{\max }-\mathrm{a}_{\min }}{s_{\mathrm{n}}}\right)$

The variable amax refers to the pixel value with maximum intensity while amin refers to the pixel value with minimum intensity in the image of interest. Variable $\mathrm{sn}_{\mathrm{n}}$ is the standard deviation of the noise defined as

$$
\mathrm{S}_{\mathrm{n}}=\sqrt{\left(\frac{1}{\Lambda-1}\right.} \sum_{(\mathrm{m}, \mathrm{n}) \in \mathrm{R}}\left(\mathrm{a}[\mathrm{m}, \mathrm{n}]-\mathrm{m}_{\mathrm{a}}\right)^{2}
$$

Where $m_{a}$ is the sample mean of the pixel brightness in the region $R$ which is the entire image in all the experiments done in this thesis. The parameter $\Lambda$ refers to the number of pixels in the region $\mathrm{R}$ and $\mathrm{a}[\mathrm{m}, \mathrm{n}]$ is the pixel value. Sample mean is computed as

$$
\mathrm{m}_{\mathrm{a}}=\frac{1}{\Lambda} \sum_{(\mathrm{m}, \mathrm{n}) \in \mathrm{R}} \mathrm{a}[\mathrm{m}, \mathrm{n}]
$$


Filtering using Pseudo Inverse Filter, Weiner Filter\& Hybrid Filter:

A sample image is taken $\&$ is subjected to salt \& Pepper noise \& guassian noise .Afterwards it shall go through filtering using Pseudo Inverse Filter, Weiner Filter\& Hybrid Filter. SNR is then computed \& compared for these approaches.

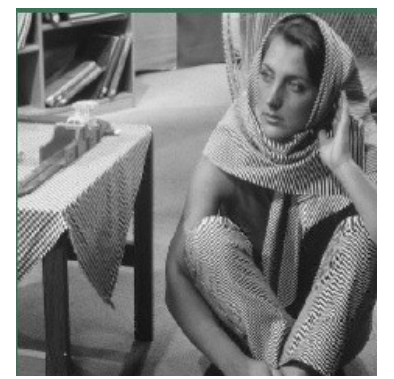

Fig 5.1 Sample Image

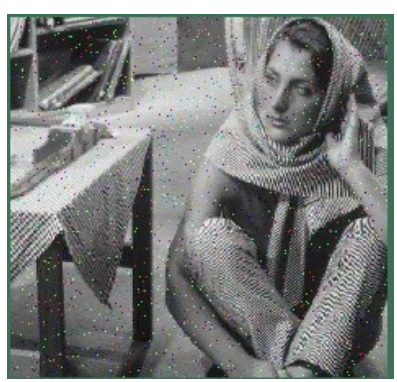

Fig 5.2 Sample Image affected by Salt-n-pepper noise

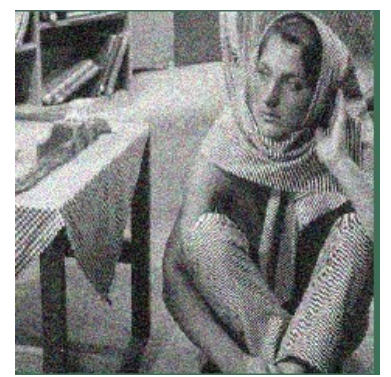

Fig 5.3 Sample Image affected by Gaussian noise

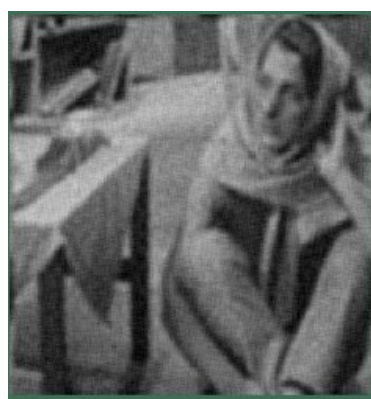

Fig 5.4 Noisy Image filtered through Pseudo Inverse Filter

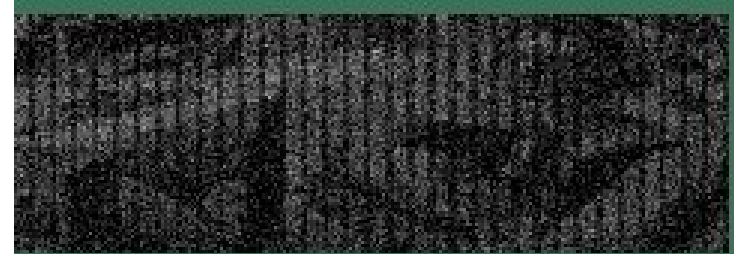

Fig 5.5 Noisy image filtered through Wiener Filter 


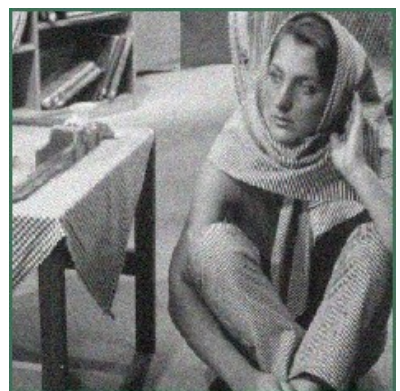

Fig 5.6 Noisy image filtered through Hybrid Filter

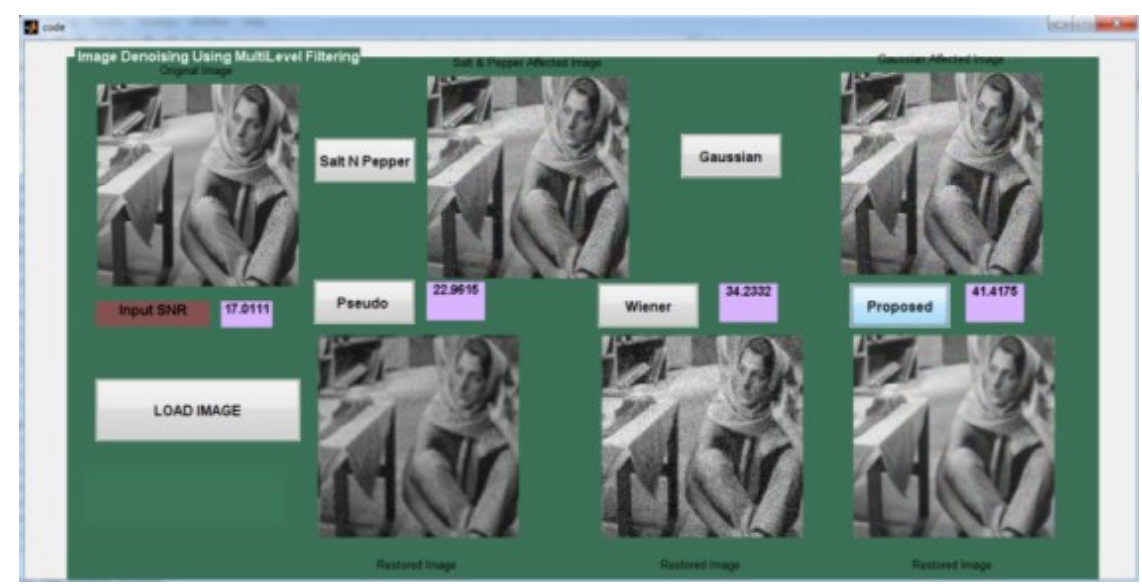

Fig 5.7 Typical view of GUI of Hybrid Filter

\begin{tabular}{|c|c|c|}
\hline METHODS & SNR OUTPUT & $\begin{array}{l}\text { NOISE, } \\
\text { V AKLANLE }\end{array}$ \\
\hline Posudu Inver $x=$ Filie & 27.43 & $\begin{array}{l}\text { 3ati and Reppe- } \\
0.05\end{array}$ \\
\hline Psevdo Inverse Filter & 21.24 & Gavssian-0.05 \\
\hline Wiener Fitrer & 47.97 & $\begin{array}{l}\text { Salt and Pepper- } \\
0.05\end{array}$ \\
\hline Wiener Flter & 22.79 & Gavssian-0.05 \\
\hline Hybridized FIL TER & 49.67 & $\begin{array}{l}\text { Salt and Pepper- } \\
0.05\end{array}$ \\
\hline Hybridized FIL TER & 26.28 & Gaussian- 0.05 \\
\hline
\end{tabular}

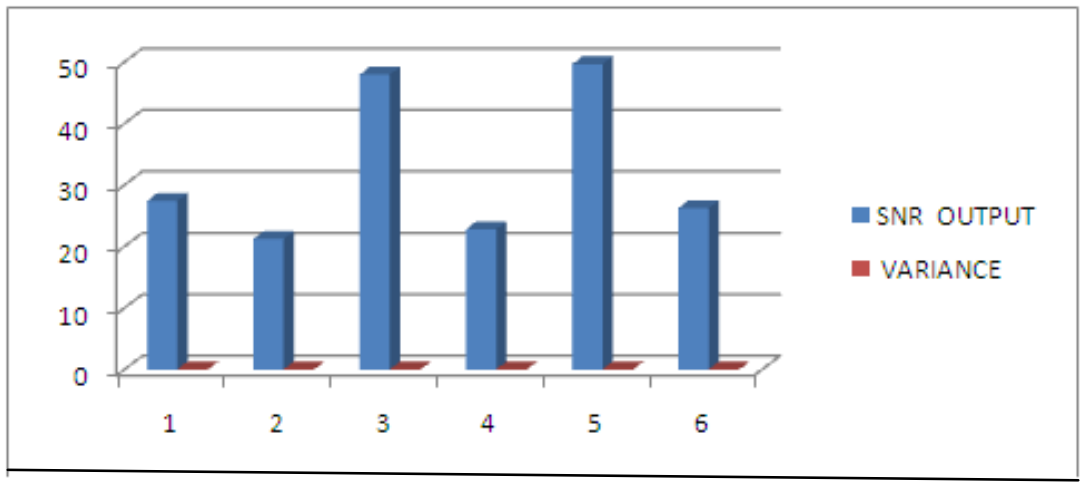

Fig 5.24 Bar chart between SNR values of various filter 


\section{Conclusion}

Since selection of the right denoising procedure plays a major role, it is important to experiment and compare the methods. As future research, we would like to work further on the comparison of the denoising techniques. If the features of the denoised signal are fed into a neural network pattern recognizer, then the rate of successful classification should determine the ultimate measure by which to compare various denoising procedures [13]. Besides, the complexity of the algorithms can be measured according to the CPU computing time flops. This can produce a time complexity standard for each algorithm. These two points would be considered as an extension to the present work done.

\section{References}

[1]. Castleman Kenneth R,'Digital Image Processing”, Prentice Hall, New Jersey, 1979

[2]. Matlab 6.1,'Image Processing Toolbox" http://www.mathworks.com/access/helpdesk/help/toolbox/images/images.shtml

[3]. Yousef Hawwar and Ali Reza, "Spatially Adaptive Multiplicative Noise Image Denoising Technique", IEEE Transaction On Image Processing, December 2002, Vol.11, No. 12.

[4]. Matlab 6.1, "Matlab", http://www.mathworks.com/, May 2001.

[5]. Matlab 6.1, "Matlab", http://www.mathworks.com/, May 2001.

[6]. B.Asad, et.al, "An Analytical Method to Compare Image Processing Filters for 3D Reconstruction of Ultrasonic Images", Proceedings of the 2007 IEEE International Conference on Mechatronics and Automation August 5 - 8, 2007, Harbin, China.

[7]. Image Processing Fundamentals-Statistics, "Signal to Noise ratio http://www.ph.tn.tudelft.nl/courses/FIP/noframes/fip-Statisti.html 2001.

[8]. Govindaraj.V, Sengottaiyan.G, "Survey of Image Denoising using Different Filters", International Journal of Science, Engineering and Technology Research (IJSETR), February 2013 ,Vol.2, Issue- 2 . 